\title{
Variabilité diatopique des associations évoquées par les mots en Francophonie
}

\author{
Michèle Debrenne \\ Université nationale de recherche de Novossibirsk, Russie \\ micheledebrenne@gmail.com
}

\begin{abstract}
Résumé. La communication portera sur l'analyse des résultats d'une étude psycholinguistique de fixation de la première réaction à un stimulus donné à partir d'une liste de 100 mots courants en France métropolitaine, Belgique wallone, Suisse romande et Canada francophone. Pour ces quatre zones seront comparées les réponses les plus fréquentes, le contenu des champs associatifs et l'ensemble des grands connecteurs structurant le réseau lexical figuré dans notre corpus en un «petit monde ». L'ensemble de ces données permettra de mesurer d'une manière objective les différences et les ressemblances profondes des diverses variétés du français au niveau du lexique et des connotations évoquées par les mots.
\end{abstract}

\begin{abstract}
In order to verify the hypothesis of the existence of a link between the "vision of the world" and language, we propose to compare word associations given by native speakers of French in different countries (France, Belgium, Switzerland, Canada). A psycholinguistic free association test was used, where about 500 responses from each zone were collected to 100 stimulus each (frequent nouns, verbs, adjectives, adverbs). A comparative analysis of the collected data shows that the most frequent reaction is mostly the same in the four francophone regions, especially when those reactions are highly popular. A comparison of the coincidences and discrepancies of the reactions to the stimulus mer (sea), homme (man), porter (to bring) is given, showing a big amount of different individual reactions. In the same time the number of different common reactions in not so big, but they are very frequent and represent about $75 \%$ of all reactions. A deeper analysis of association fields with four different approaches tends to prove that their structure is similar, but the concrete lexical content of different subcategories can differ from one language to the other. The structure of the lexicon of French speaking subject of the experience is a "small world" with the same set of superconnectors. In conclusion we make the supposition that dictionaries of word associations can display how language reflects a "vision of the world", and this vision does not depend on the socio-cultural environment but mostly on the lexicon itself.
\end{abstract}




\section{L'hypothèse de départ}

Depuis Humboldt, Sapir et Whorf, les linguistes sont partagés sur la nature des relations entre la langue et la pensée, tout à tour approuvant ou niant l'existence d'une "vision du monde" reflétée par les mots. Ce qui semble évident au linguiste naïf (la "saudade" portugaise ne correspond pas plus à "nostalgie" que le "spleen" anglais) ne l'est plus au chercheur féru d'universalisme. On retrouve ici, sous un autre angle, le célèbre paradoxe de la traduction (rien n'est véritablement traduisible à l'identique, et pourtant les gens se comprennent entre eux et on traduit - avec plus ou moins de succès - qu'il s'agisse d'œuvres littéraires que de modes d'emploi de machines à laver).

Il existe différentes méthodes d'étude des "points de vue" sous-tendus par les mots, ces connotations partagées par les locuteurs d'une langue que les dictionnaires classiques ne retiennent pas toujours (Raccah, 2014). On peut, par exemple, analyser les métaphores (Underhill, 2012). L'introspection linguistique des bilingues est parfois utile, mais souvent peu fiable. On peut également appliquer la méthode psycholinguistique de fixation de la première réponse à un stimulus lexical et créer ainsi ce qu'on appelle des "dictionnaires d'associations évoquées par les mots" (cf. par ex. Nelson et al, 1998)

C'est notre approche, et dans cette étude nous nous proposons de comparer les réactions des différents locuteurs du français dans quatre zones de la francophonie, soit pour l'instant: la France métropolitaine, la Wallonie et le Canada francophone. Les données pour la Suisse romande n'ayant pas été pour l'instant réunies en nombre suffisant (qui est de 500 minimum), les résultats pour cette zone ne présentent pas un caractère définitif et seront fournies à titre indicatif. Le but principal cette étude est de mettre en évidence ce qui est commun aux locuteurs d'une même langue et ce qui ne l'est pas, d'apprécier à quel point une communauté linguistique partage une même "vision du monde" ou si cette dernière est une vue de l'esprit. En effet, si les connotations contenues dans les mots et mises en évidence par l'expérience sont les mêmes pour les locuteurs du français à Paris et à Montréal, on peut en déduire qu'elles sont liées à la langue partagée et non pas aux circonstances sociolinguistique de sa pratique.

Dans ce qui va suivre nous analyserons les résultats d'une étude psycholinguistique de fixation de la première réaction à un stimulus donné à partir d'une liste de 100 mots courants. Pour les quatre zones de la Francophonie prises en considération pour l'instant, nous présenterons la comparaison des réponses les plus fréquentes, le contenu des champs associatifs et l'ensemble des grands connecteurs structurant le réseau lexical figuré dans notre corpus en un «petit monde ». L'ensemble de ces données sera publié sous la forme d'un Dictionnaire électronique des normes associatives de la Francophonie (infra DENAF) qui permettra de mesurer d'une manière objective les différences et les ressemblances profondes des diverses variétés du français.

\section{La méthode}

\subsection{Les dictionnaires d'associations évoquées par les mots}

Un dictionnaire d'associations évoquées par les mots (dites aussi verbales ou lexicales) est le résultat d'une expérience psycholinguistique de fixation de la première réaction à un stimulus (un mot de la langue) proposé à un locuteur ordinaire. A l'heure actuelle il en existe un certain nombre, pour des langues telles que l'anglais (voir deux projets sur internet, celui de G. Kiss pour l'anglais et celui de D.L. Nelson pour l'américain), le russe (notamment Караулов et al 1994-1998, Гуц, 2004, Стернин 2011, Голдин 2009), le français (Дебренн, 2010a et b). Un intéressant projet de l’Université de Liège «A small 
world of words » propose une approche similaire pour plusieurs langues (anglais, allemand, néerlandais, portugais, espagnol, chinois, japonais, vietnamien).

Un dictionnaire d'associations comporte deux parties : d'une part le dictionnaire direct, formé de la liste de mots-vedettes et des réponses données par les personnes interrogées (classées par ordre de fréquence décroissante) et le dictionnaire inverse, présentant cette fois ces réactions, accompagnées des stimulus qui les ont provoquées (là aussi, en ordre décroissant de fréquence). Ces champs associatifs ainsi représentés diffèrent de ce qu'on trouve dans les dictionnaires classiques :

- Les dictionnaires ordinaires sont le fruit de l'activité lexicographique d'une personne ou d'un groupe de personnes sur la base de leurs connaissances personnelles établies au cours de l'analyse d'un certain nombre de contextes, tandis que chaque lexème d'un dictionnaire d'associations est présenté à 500 personnes.

- Les dictionnaires d'associations contiennent les connaissances conscientes, des connaissances semi-conscientes et des connaissances inconscientes.

- Ils contiennent une partie représentée par des connaissances individuelles, obtenues par des réponses non répétitives, changeantes dans le temps, et une partie générale, constante, dans laquelle sont réunies les connaissances partagées par tous : les expériences menées depuis près de 50 ans par les chercheurs russes permettent de mettre en évidence ce « noyau dur » des normes associatives.

\subsection{L'expérience}

L'expérience qui a permis de réunir la matière pour le $D E N A F$ s'est déroulée sur internet du 15 septembre 2013 au 15 septembre 2015. Pour cela il a été proposé par l'intermédiaire d'un questionnaire Google sur à des locuteurs du français de France, Belgique, Suisse et Canada de participer à une expérience de fixation de la première réaction à une liste de 100 mots, identiques pour tous. Ces mots font partie des plus courants de la langue française, selon les données du site http://eduscol.education.fr. Il faut reconnaitre que les dictionnaires de fréquence pour le français ne sont pas très répandus ni modernes (Henmon 1924, Juilland et al, 1970). Par la suite nous avons pu constater que les mots proposés en tant que stimuli ne sont, pour les trois quarts de la liste, parmi les 500 plus fréquents mots du dictionnaire de Lonsdale et LeBras (2009). Cependant certains lexèmes (frère, regard, ciel, mari, âme, soleil, Dieu) y figurent avec des scores relativement bas. Etant donné le but de l'étude, qui est de comparer les normes d'association (et non pas ces normes elles-mêmes) on peut considérer que ces différences de fréquences n'ont pas beaucoup d'importance.

Nous avons également évité d'utiliser des stimuli plurisémantiques qui dans certains cas peuvent porter à confusion (par ex. fin qui peut être interprété par les participants à l'expérience comme un adjectif masculin ou comme un substantif, ou marche comme un substantif, par ailleurs plurisémantique, ou une forme verbale). Dans le cas de bien, que nous avons conservé car il s'agit d'un mot très fréquent de la langue, nous n'avons pas cru bon de préciser s'il s'agissait de l'adverbe ou du substantif. Enfin, nous avons introduit quelques mots qu'il nous semblait intéressant de comparer dans différentes variations de la francophonie malgré qu'ils ne soient pas d'une grande fréquence, comme, par exemple, mer.

Afin d'éviter le voisinage de mots sémantiquement proches dans la liste, ainsi que le caractère artificiel de l'ordre alphabétique, nous avons modifié l'ordre dans lequel ils apparaissent dans l'expérience pour finalement obtenir la liste suivante : mer, parler, main, bien, être, temps, regarder, mari, faire, heure, venir, fois, femme, avoir, monde, enfant, répondre, fille, petit, vouloir, mal, appeler, ville, pied, dire, homme, rester, beau, vivre, 
frère, ciel, pouvoir, jour, yeux, jeune, grand, aller, vie, voir, chose, devoir, prendre, tête, trouver, père, donner, moment, savoir, bon, passer, terre, aimer, an, arriver, dieu, penser, maison, croire, coup, demander, cœur, entendre, nuit, connaître, sortir, voix, air, devenir, mot, sentir, vieux, comprendre, blanc, corps, soleil, partir, soir, esprit, rue, sembler, ami, noir, nouveau, mourir, reprendre, état, mère, entrer, pays, tenir, mort, regard, gens, revenir, amour, rendre, attendre, âme, porter, chercher.

La méthode employée pour réunir la matière a été la même pour les quatre sous-ensembles: à intervalles réguliers un message électronique a été envoyé vers une centaine d'adresses prises sur des sites divers, en général sur ceux d'universités francophones ou d'organismes liés à l'enseignement. Un site internet a également été créé, sur lequel les destinataires du message peuvent en apprendre plus sur l'expérience, les travaux précédents et leurs résultats. Les résultats étaient collectés dans un fichier Excel. Malgré que cette méthode de collecte ait été strictement identique pour les quatre zones, les réponses sont arrivées dans une proportion très différente depuis chaque pays : le nombre minimal (fixé à 500 , en se basant sur les travaux de Cherkassova (Черкасова 2006)) a été atteint en France (notée F dans la suite de cet article) au bout de six mois. Depuis ce chiffre a continué de croître sans actions supplémentaires de notre part, pour atteindre 604 réponses valables à la fin de l'expérience. A cette date, deux ans après le début de l'expérience, les 500 réponses requises étaient atteintes pour la Belgique et le Canada, (resp. B et C) mais seules 326 réponses avaient été obtenues de Suisse romande (S). Nous n'avons pas d'explication à cette différence significative dans le fonctionnement de notre système de récolte de la matière.

Le formulaire de réponse contenait également une partie informative, très succincte, avec des questions sur le sexe, l'âge, le pays et la ville où habite la personne participant au test, ainsi que sa profession. La première question était la suivante «le français est-il votre langue maternelle ? (oui/non)». Etant donné que ces questions apparaissaient en dernier, nous n'avons pris en compte que les formulaires dans lesquels ces questions étaient renseignées. Nous estimons à environ $5 \%$ le nombre de formulaires rejetés pour cette raison.

\subsection{La méthode d'analyse}

La première étape du traitement des données consiste en sa vérification. La première tâche consiste à éliminer les formulaires incomplets (ceux dans lesquels la partie informative n'a pas été renseignée) ainsi que les réponses contenant plus d'une association, par ex. doigt, fabrication, montrer en réaction à main, ce qui n'est pas une erreur en soi mais ne correspond pas aux conditions de notre expérience de fixation de la première réponse (des réponses sous forme de syntagmes ou courtes phrases sont cependant admises). Nous nous sommes également efforcés d'unifier l'emploi des articles dans les réactions : ainsi, les réponses la main dans la main ont été corrigées en main dans la main (plus fréquentes) afin qu'elles soient comptabilisées ensemble.

Après une première étape semi-automatique de vérification orthographique pour unifier l'utilisation des majuscules (due généralement à l'utilisation par les participants à l'expérience de gadgets électroniques réglés sur l'emploi d'une majuscule à chaque nouvelle entrée) et rectifier les fautes d'orthographe lexicales les plus évidentes (signes diacritiques absents ou superflus, problèmes sur les lettres doubles) qui représentent l'immense majorité des erreurs rencontrées, une vérification manuelle s'impose, pour les erreurs d'orthographe grammaticale. En effet en l'absence de contexte seul l'opérateur humain peut déterminer l'erreur dans doit en réaction à main ou, au contraire, décider de ne pas corriger home en homme dans le cas d'une réaction à maison. C'est l'ensemble des 
réactions pour le stimulus donné qui doit être pris en compte pour faire des choix. Dans le premier cas, c'est la fréquence minimale de la réaction doit (trois en tout pour ce stimulus) confrontée à la popularité de doigt (deuxième en fréquence pour ce stimulus toutes zones confondues) qui nous a poussé à choisir cette seconde graphie. Il convient de reconnaître que dans l'ensemble la correction orthographique de la matière réunie pour ce dictionnaire est notablement plus élevée que celle que nous avions constatée lors de nos expériences précédentes. Là encore nous n'avons pas d'explication toute prête à ce phénomène, si ce n'est que dans le cas du DENAF nous ne nous sommes pas limités à des étudiants, le public concerné va de 10 à 83 ans. Il se peut également que cela soit dû à l'utilisation de systèmes de correction automatique par les participants.

La structure classique d'un article de tout dictionnaire direct d'associations a la forme d'une hyperbole : sur 500 réactions, quelques-unes sont partagées par un grand nombre, puis cette courbe chute pour montrer un grand nombre de réactions individuelles, comme on peut le voir dans l'article suivant, formé des réponses des francophones de la France métropolitaine ayant participé à l'expérience:

Ami : ennemi 68; copain 60; fidèle 26; proche 21; confiance 20; frère 19; cher 17; confident 15; amour, meilleur 14; amie 13; famille, rare 10; amitié, pote 8; camarade, vie 7; fidélité, important, soutien 6; chien, intime, précieux 5; aide, aimer, compagnon, de toujours, lien, pour la vie, toujours, vrai 4; besoin, de cœur, d'enfance, échange, friend, indispensable, loyauté, partage, petit, sincérité 3; amant, ami-ami, amical, bien, chaleur, chaud, choisi, confidence, connaissance, éternel, fête, fraternité, oui, peu, rire, sûr, toi, véritable, voisin 2; affinités, allié, beau, bienveillance, bon temps, camaraderie, cocu, cœur, collègue, comme des frères, compagnon de route, complice, complicité, convivialité, de 30 ans, de bien, déçue, de longue date, de ta femme, devenir, distance, doigts de la main, don, d'un soir, durée, embrassades, enfance, enfant, entends-tu le vol noir du corbeau, épaule, époux, être cher, ex-meilleur ami, faux, faux ami, fille, fréquentations, Gary, gens, groupe, homme, jeux, joie, lien connu, lointain, longue histoire, main, moitié, nature, Nicolas, parler, partager, parti, pauvre, personne proche, personne très proche, plus que copain, présent, primordial, priorité, quelqu'un, rareté, réconfort, relatif, relation, retrouvé, rien de plus cher, se confier, sentiments, sincère, si rare, solidaire, sortie, sur qui on peut compter, sympathie, tendresse, ton, trahison, très cher, trompeur, union, unique, valeur sure, vérité, vieux, visage souriant, vital, Willy $1 .(587,148,29,88)$

Les chiffres indiqués entre parenthèses indiquent respectivement le nombre de réponses obtenues à ce stimulus en particulier, le nombre de réponses différentes, celui des refus de réponses et celui des hapax.

Dans le cas du dictionnaire inverse, les articles sont formés des réactions données aux stimuli proposés lors de l'expérience d'association libre. Les entrées des articles sont suivies des stimuli qui les ont provoquées, disposés par ordre décroissant de fréquence. Le chiffre indique le nombre de personnes ayant répondu par cette réaction à ce stimulus. A l'intérieur d'un groupe de fréquence identique, les stimuli sont donnés par ordre alphabétique. Ainsi, l'article suivant (tiré du même corpus):

Amour : cœur 87; aimer 67; mère 17; mari 16; ami 14; Dieu 7; chercher, enfant, faire, frère 6; femme, regard 5; père 4; amour, grand, trouver 3; beau, donner, moment, mot, rendre, vie 2 ; fille, homme, mal, mort, rester $1 .(272,27)$

indique signifie que la réaction amour a été donnée 87 fois au stimulus cœur, 17 fois à mère et 16 fois à mari, etc. A la fin de l'article sont indiqués deux chiffres entre parenthèses : il s'agit du nombre total d'apparition de ce lexème en tant que réaction dans le corpus (272 dans le cas de amour) et nombre de stimuli ayant provoqué cette réaction (27). 
Si l'on peut se contenter de présenter parallèlement les données obtenues dans les quatre zones géographiques étudiées, comme cela a été fait pour le dictionnaire des associations des langues slaves (Уфимцева et al. 2004) pour le russe, biélorusse, bulgare et l'ukrainien, il nous a cependant semblé plus intéressant d'analyser certaines données plus en profondeur.

Ainsi nous avons analysé, pour chaque stimulus, chaque zone et en comparaison avec l'ensemble du corpus :

- Les réactions les plus fréquentes, et surtout la première, la fréquence de cette réponse (en nombre absolu et en pourcentage),

- Les réactions communes aux différentes zones et leur nombre (ainsi que le nombre de réactions différentes et leur quantité absolue). Il est aisé de calculer qu'on peut théoriquement obtenir les sous-ensembles suivants FBSC, FBS, FSC, FBC, BSC, FB, FS, FC, BS, BC, SC, F, B, S et C. Ces données ont également été analysées pour l'ensemble du corpus (et non pas pour un stimulus pris à part).

- Le contenu sémantique des champs associatifs (l'ensemble des réactions à un stimulus donné) suivant différentes méthodes.

De même, dans le dictionnaire inverse, pour chaque réaction, chaque zone et l'ensemble du corpus nous avons comparé les stimuli qui l'ont provoquée et leur nombre. Nous avons en particulier analysé pour chaque zone géographique les réactions les plus fréquentes, ainsi que celles qui ont été provoquées par le plus grand nombre de stimuli. Ces dernières sont les superconnecteurs des réseaux lexicaux et leur confèrent ce caractère dit de «petit monde » (Solé, 2005).

\section{Les résultats}

Nous présentons ici les premiers résultats de cette étude, en rappelant que les données pour la Suisse ne sont pas définitives.

\subsection{Les réactions les plus fréquentes}

\subsubsection{La première réaction}

Il a été remarqué lors de la création d'autres dictionnaires d'associations que la réaction la plus fréquente apparait très tôt, dès que le nombre de réponses atteint 200. Dans notre corpus, cette première réaction est la même dans $62 \%$ des cas pour les quatre sousensembles, surtout, mais pas toujours, quand le pourcentage de cette réponse parmi les autres réactions données à ce stimulus est élevé (voir Tableau №1). 
Tableau №1 : Pourcentage de la première réaction pour les stimuli penser, jeune, cœur.

\begin{tabular}{|c|c|c|c|c|c|c|c|c|c|}
\hline \multirow[t]{2}{*}{ stimulus } & \multirow{2}{*}{$\begin{array}{l}\text { première } \\
\text { réaction } \\
\text { commune } \\
\text { FBSC }\end{array}$} & \multicolumn{8}{|c|}{ \% de cette réaction parmi les réponses } \\
\hline & & \multicolumn{2}{|c|}{$\mathrm{F}$} & \multicolumn{2}{|c|}{$\mathrm{B}$} & \multicolumn{2}{|c|}{$\mathrm{S}$} & \multicolumn{2}{|c|}{$\mathrm{C}$} \\
\hline penser & réfléchir & 0,62 & 15,4 & 0,83 & 44,7 & 1,19 & 41,8 & 0,76 & 37,7 \\
\hline jeune & vieux & 0,72 & 45,9 & 0,94 & 51,2 & 1,23 & 55,9 & 0,89 & 35,5 \\
\hline coeur & amour & 0,58 & 15,2 & 0,84 & 21,5 & 0,94 & 15,7 & 0,72 & 15,2 \\
\hline
\end{tabular}

Dans ce tableau nous voyons deux données chiffrées. La première indique le pourcentage occupé par cette réaction par rapport au nombre de réactions différentes données pour ce stimulus. La seconde est le rapport ramené à 100 du nombre d'occurrences de cette réaction au nombre total de réponses données à ce stimulus. Ces deux chiffres sont liés, mais pas directement. En effet, un premier chiffre élevé indique que ce stimulus n'a pas provoqué une grande variété de réactions, mais pas obligatoirement que la première d'entre elle est très populaire, auquel cas le deuxième chiffre le sera aussi. Ainsi, la réaction amour à cœur en Suisse présente un pourcentage plus élevé que dans les autres zones, mais c'est parce que ce stimulus n'a donné que 106 réactions différentes (cf. 171 en France, 137 au Canada etc.). Par contre le stimulus jeune n'a pas donné une grande variété de réactions (avec un minimum de 81 en Suisse), et la première réaction, vieux est, de plus, unanimement populaire : en Suisse et en Belgique plus de la moitié des interviewés l'ont proposée.

On observe les coïncidences suivantes des premières réactions entre les différentes zones (pour 100 stimuli) :

Tableau №2 : Proportion des coïncidences de la première réaction

\begin{tabular}{|l|l|l|l|l|l|l|l|l|}
\hline FBSC & FSB/C & FBC/S & BSC/F & FSC/B & FC/BS & BS/F/C & FC/B/S & F/B/S/C \\
\hline 62 & 12 & 8 & 5 & 2 & 5 & 3 & 2 & 1 \\
\hline
\end{tabular}

Il est intéressant de remarquer que le pourcentage de réponses identiques a tendance à augmenter avec le nombre de participants. Au bout d'un an de récolte du matériel, pour une quantité de réponses de $\mathrm{F}=500, \mathrm{~B}=186, \mathrm{~S}=124$ et $\mathrm{C}=114$ nous ne notions que $53 \%$ de premières réponses identiques.

Dans le cas où la première réaction n'est pas la même dans les quatre pays, les réponses différentes sont en général celles qui sont données en deuxième ou troisième position par les interviewés des autres pays (voir Tableau №3). Par exemple, esprit comme réponse à âme, donné en première réponse par les interviewés français, belges et suisses, est la deuxième réponse pour ceux du Canada.

Tableau №3. Comparaison des premières réponses pour les stimuli âme, amour, main. Les pourcentages indiqués sont ceux du nombre d'occurrences de cette réaction au nombre total de réponses données à ce stimulus.

\begin{tabular}{|l|l|l|l|l|}
\hline stimulus & F & B & S & C \\
\hline Âme & esprit $24,6 \%$ & esprit $24,6 \%$ & esprit $20,7 \%$ & saur $24,1 \%$ \\
\hline Amour & toujours $32,4 \%$ & toujours $16,3 \%$ & toujours $16,4 \%$ & haine $8,7 \%$ \\
\hline Air & respirer $15,1 \%$ & respirer $9,5 \%$ & respirer $14,1 \%$ & pur $13,4 \%$ \\
\hline
\end{tabular}

Notons que le stimulus amour n'a évoqué toujours comme réaction qu'auprès de seize Canadiens (sur 424). 


\subsubsection{Les sept réactions les plus fréquentes}

Ces premiers résultats tendraient à montrer qu'il n'y a pas de différences significatives dans les premières réactions parmi les locuteurs des quatre pays étudiés. Cela n'est pas très étonnant, puisque la première réaction est par définition la plus standardisée. Cependant nous remarquons que les cas où les réactions en provenance du Canada sont différentes à celles du continent sont plus fréquents que les autres. Cette impression est renforcée par le fait que fréquemment, même dans le cas où les réactions sont identiques dans les quatre zones, le pourcentage que cette réaction représente est nettement inférieur au Canada que dans les trois autres zones. Dans le tableau ci-dessous (Tableau №4) on remarque en effet ce phénomène, qui reflète que les participants à l'expérience du Canada ont une réaction moins standardisée que celles des autres pays.

Tableau №4. Comparaison du pourcentage de la première réaction. Les pourcentages indiqués ici sont ceux du nombre d'occurrences de cette réaction au nombre total de réponses données à ce stimulus.

\begin{tabular}{|l|l|l|l|l|l|}
\hline Stimulus & $\begin{array}{l}\text { première } \\
\text { réaction }\end{array}$ & F & B & S & C \\
\hline Prendre & donner & 11,3 & 16,3 & 15,6 & 10,5 \\
\hline Regarder & voir & 52,3 & 50,8 & 47,5 & 41,8 \\
\hline Fille & garçon & 50,2 & 55,5 & 54,1 & 36,5 \\
\hline
\end{tabular}

Il peut cependant être intéressant de comparer, non seulement la première, mais les dix premières réactions à chaque stimulus. En effet, la particularité de la répartition en fréquence des réactions dans une expérience d'associations évoquées par les mots est telle que la somme des occurrences des cinq premières réactions représente entre un tiers et la moitié de toutes les réactions. Comme on le voit dans le tableau ci-dessous pour le stimulus main (Tableau №5), les 7 premières réactions coïncident pratiquement (sauf bras, absent des 10 premières réponses au Canada, où il est en onzième position) dans les quatre zones observées, avec toutefois des pourcentages variables :

Tableau №5. Comparaison des dix premières réactions au stimulus main. Les réactions communes aux quatre zones géographiques sont en italiques gras.

\begin{tabular}{|l|l|l|l|l|l|l|l|l|}
\hline$№$ & $\mathrm{~F}$ & & $\mathrm{~B}$ & & $\mathrm{~S}$ & & $\mathrm{C}$ & \\
\hline 1 & pied & 25.3 & pied & 28,5 & pied & 35,6 & pied & 18,3 \\
\hline 2 & doigt & 13.7 & doigt & 16,5 & doigt & 13,3 & doigt & 13,9 \\
\hline 3 & doigts & 7,3 & doigts & 9,3 & doigts & 6,5 & droite & 8,1 \\
\hline 4 & toucher & 3,6 & bras & 2,9 & droite & 3,2 & doigts & 7,1 \\
\hline 5 & bras & 3,2 & toucher & 2,4 & bras & 2,9 & toucher & \\
\hline 6 & droite & 2,9 & $\begin{array}{l}\text { gauche } \\
\text { serrer }\end{array}$ & 2 & tendue & 1,9 & gauche & 2,7 \\
\hline 7 & $\begin{array}{l}\text { gauche } \\
\text { prendre }\end{array}$ & 2,2 & $\begin{array}{l}\text { droite } \\
\text { écrire }\end{array}$ & 1,7 & $\begin{array}{l}\text { bague, } \\
\text { gauche }\end{array}$ & 1,6 & pieds & 2 \\
\hline
\end{tabular}


Cette étude devra être poursuivie pour tous les stimuli et l'ensemble du corpus. Ci-dessous nous proposons une analyse non plus seulement quantitative, mais également qualitative des sous-ensembles des réactions obtenues pour chaque stimulus dans les quatre zones étudiées.

\subsubsection{Les champs associatifs}

On appelle «champ associatif » l'ensemble des réactions obtenues à un stimulus. La comparaison des champs associatifs passe d'une part par l'étude approfondie des intersections entre les sous-ensembles de réactions et par l'analyse sémantique des réactions.

Nous nous proposons de comparer les coïncidences et divergences dans l'ensemble des réactions formant le champ associatif de trois stimuli, mer, homme et porter. Mer a été choisi parce qu'il est le premier dans la liste des stimuli, les stimuli précédents n'ont pas engendré d'associations supplémentaires et l'enthousiasme est intact chez les interviewés. De plus on peut supposer a priori que Français, Belges, Suisses et Canadiens auront une vision différente de la mer, en raison des conditions géographiques qui caractérisent ces différents pays. Au contraire, homme est une notion universelle, ce substantif se trouve vers le milieu de la liste. Quant à porter, il est placé vers la fin, alors qu'une certaine lassitude se fait sentir chez les interviewés (tous ne vont pas jusqu'au bout) et qu'ils ont tendance à réfléchir moins longtemps, à inscrire vraiment la première chose qui leur vient à l'esprit, ce qui est particulièrement précieux pour nous. Par ailleurs l'analyse de l'ensemble du champ associatif pour ce stimulus n'a pas mis en évidence de « leader » particulier, mais beaucoup de réponses avec un score moyen. Les quinze possibilités d'intersections sont représentées dans le tableau suivant (Tableau №6):

Tableau № 6. Coïncidence des réactions dans les différentes zones étudiées pour les stimuli mer, homme, porter. ${ }^{*}$ - pourcentage de ces réactions sur l'ensemble des réactions différentes pour ce stimulus sur l'ensemble du corpus, ${ }^{* *}$ - pourcentage du total des réactions de cette catégorie par rapport à l'ensemble de toutes les réactions à ce stimulus sur l'ensemble du corpus.

\begin{tabular}{|c|c|c|c|c|c|c|c|c|c|c|c|c|}
\hline & \multicolumn{4}{|c|}{ Mer } & \multicolumn{4}{|c|}{ homme } & \multicolumn{4}{|c|}{ porter } \\
\hline & \multicolumn{2}{|c|}{$\begin{array}{l}\text { réactions } \\
\text { différentes }\end{array}$} & \multicolumn{2}{|c|}{$\begin{array}{l}\text { total des } \\
\text { réactions }\end{array}$} & \multicolumn{2}{|c|}{$\begin{array}{l}\text { réactions } \\
\text { différentes }\end{array}$} & \multicolumn{2}{|c|}{$\begin{array}{l}\text { total des } \\
\text { réactions }\end{array}$} & \multicolumn{2}{|c|}{$\begin{array}{l}\text { réactions } \\
\text { différentes }\end{array}$} & \multicolumn{2}{|c|}{$\begin{array}{l}\text { total des } \\
\text { réactions }\end{array}$} \\
\hline & $\mathrm{nb}$ & $\% *$ & $\mathrm{nb}$ & $\% * *$ & $\mathrm{nb}$ & $\%$ & $\mathrm{nb}$ & $\%$ & nb & $\%$ & $\mathrm{nb}$ & $\%$ \\
\hline FBSC & 27 & 10,47 & 1295 & 75,12 & 13 & 4,64 & 1255 & 73,48 & 37 & 11,28 & 1143 & 67,75 \\
\hline FBS & 5 & 1,94 & 33 & 1,91 & 5 & 1,79 & 24 & 1,41 & 6 & 1,83 & 42 & 2,49 \\
\hline FSC & 7 & 2,71 & 34 & 1,97 & 8 & 2,86 & 38 & 2,22 & 8 & 2,44 & 48 & 2,85 \\
\hline $\mathrm{BSC}$ & 4 & 1,55 & 14 & 0,81 & 2 & 0,71 & 9 & 0,53 & 3 & 0,91 & 12 & 0,71 \\
\hline FBC & 5 & 1,94 & 39 & 2,26 & 7 & 2,50 & 43 & 2,52 & 15 & 4,57 & 77 & 4,56 \\
\hline FB & 12 & 4,65 & 31 & 1,80 & 9 & 3,21 & 27 & 1,58 & 9 & 2,74 & 26 & 1,54 \\
\hline FS & 6 & 2,33 & 24 & 1,39 & 6 & 2,14 & 12 & 0,70 & 11 & 3,35 & 33 & 1,96 \\
\hline $\mathrm{FC}$ & 8 & 3,10 & 28 & 1,62 & 15 & 5,36 & 49 & 2,87 & 12 & 3,66 & 32 & 1,90 \\
\hline $\mathrm{SC}$ & 7 & 2,71 & 17 & 0,99 & 3 & 1,07 & 6 & 0,35 & 6 & 1,83 & 13 & 0,77 \\
\hline BS & 3 & 1,16 & 7 & 0,41 & 2 & 0,71 & 6 & 0,35 & 2 & 0,61 & 4 & 0,24 \\
\hline $\mathrm{BC}$ & 0 & 0,00 & 0 & 0,00 & 5 & 1,79 & 12 & 0,70 & 9 & 2,74 & 19 & 1,13 \\
\hline $\mathrm{F}$ & 63 & 24,42 & 73 & 4,23 & 79 & 28,21 & 87 & 5,09 & 64 & 19,51 & 67 & 3,97 \\
\hline B & 34 & 13,18 & 38 & 2,20 & 46 & 16,43 & 50 & 2,93 & 34 & 10,37 & 38 & 2,25 \\
\hline $\mathrm{S}$ & 33 & 12,79 & 33 & 1,91 & 31 & 11,07 & 33 & 1,93 & 49 & 14,94 & 53 & 3,14 \\
\hline $\mathrm{C}$ & 44 & 17,05 & 58 & 3,36 & 50 & 17,86 & 57 & 3,34 & 63 & 19,21 & 80 & 4,74 \\
\hline
\end{tabular}


On constate le nombre de réactions différentes communes (FBSC) n'est pas très élevé, (par exemple 27 pour $m e r$, ce qui représente $10,4 \%$ de toutes les réactions différentes à ce stimulus) mais comme elles font partie des plus fréquentes, elles représentent un très grand nombre de réactions en valeur absolue (soit 1290 et $75,1 \%$ ). Ainsi, tous les participants à l'expérience ont proposé pour mer les réactions suivantes (dans des proportions diverses) : à boire, agitée, bateau, bleu, bleue, bonheur, calme, ciel, du Nord, eau, étendue, éternité, horizon, immensité, infini, liberté, Méditerranée, océan, plage, poisson, sable, sel, soleil, terre, turquoise vacances, vague, vagues.

Là encore, nous remarquons une assez grande similitude des réponses en provenance des quatre zones étudiées. Elle sera encore plus remarquable en additionnant les variantes morphologiques des réactions (bleu/bleues, vague/vagues, vêtement/vêtements etc.) que nous avons ici comptabilisées séparément, comme il est de mise d'ordinaire dans les travaux consacrés aux dictionnaires d'association.

En ce qui concerne l'analyse sémantique des champs associatifs, nous indiquerons ici quelques pistes méthodologiques, qui doivent être affinées par la suite, pour chaque stimulus ou pour un groupe de stimuli appartenant au même champs sémantique.

Pour le stimulus maison, nous avons utilisé une méthode basée sur l'utilisation d'un schéma universel très précis en dix catégories (Гольдин, $2010: 100):$ 1- Situation mettant en scène le stimulus St ; 2- liens logiques du St (supercatégorie, subdivisions, objets du même type, contraires) ; 3- parties du St ; 4- chronotope ; 5- objets relatifs ; 6- qualités, évaluations (paramétriques, matière, forme, taille, couleur, pragmatiques, esthétiques, émotionnelles, etc.) ; 7- actions, états du St ; 8- relation à l'homme (catégories d'humains liés au St, appartenance au locuteur, interaction avec le locuteur, objets fonctionnellement liés au St) ; 9- aspects discursif du lexème représentant le St (synonymes, définition métalinguistique, allusions textuelles, jeux de mots, définition) ; 10- valeur symbolique.

Notre analyse du champ associatif de maison dans les quatre sous-corpus montre que si les mêmes catégories sont présentées à chaque fois, leur contenu peut varier d'une région à l'autre. La seule catégorie vide parmi les réponses en provenance du Canada est celle des jeux de mots et assonances (alors que nous avons toison, foison ou raison ailleurs). C'est par contre le seul corpus qui propose des réactions comme portefeuille ou argent que nous avons classées - sous toutes réserves - parmi les objets fonctionnellement liées au stimulus. Seule la liste française présente une réaction «humain lié au St» (en l'occurrence architecte), seule la liste canadienne comporte une réaction hiver (4-chronotope). Dans l'ensemble les quatre sous-ensembles du champ associatif de maison sont très comparables. On remarquera (voir le tableau №7), cependant, que le corpus canadien présente une plus grande variété de réactions explicitant le rapport entre le locuteur et la maison: 
Tableau № 7. Comparaison du contenu de la catégorie 8-2« appartenance du stimulus au locuteur ». Les chiffres indiqués sont ceux du nombre d'occurrences de la réaction.

$\mathrm{F}$

famille, 17 , chezsoi, 12 ; de famille, 2 ; parents, 2 ;

soi, 2 ;

chez, 1 ;

chez soi !, 1 ;

familiale, $1 ;$ mon 3

toit, 1 ;

\section{B $\quad S$}

chez-soi, $\quad 11$; chez-soi, 15 ; chezfamille, 19 ; chez- moi, 6 ; famille, 6 ; moi, 2 ; propriété, de famille, 1 ; chez, 2 ; bien familial, $1 ; 2$; moi, 1 ; chez-toi, 1 ; nous, 1 ; parents, 1 ; la mienne 1 paternelle, 1; soi, maman, 1; soi, 1 ;

\section{C}

chez-soi, 20 ; famille, 16 ; chezmoi, 6; familiale, 8 ; paternel ; 3 ; chez nous , 2 ; nous, 2 ; possession, 2 ; ma, 1 ; ma maison, 1 ; maman, 1 ; parent, 1 ; parents, 1 ; propriétaire, 1 ; soi, 1 ;

Une analyse comparative détaillée du contenu de chaque sous-catégorie permettra de faire des conclusions sur les ressemblances et différences de l'image évoquée par chaque stimulus dans les régions étudiées.

Pour le stimulus passer, nous proposons la méthode dite de l'analyse de la signification psychologique (Стернин, Рудакова 2011 : 139) qui préconise de délimiter dans le champ associatif autant de zones de réactions qu'il y a de significations différentes du lexème. Pour passer nous avons défini les significations suivantes : passer = mouvement (en particulier «mouvement le long de qqch », « mouvement rapide », « ce qui peut passer », « mouvement répété ») ; passer = franchir, traverser (en particulier « doubler, dépasser»; passer $=$ continuer sans s'arrêter $;$ passer $=$ changer d'activité (de thème de conversation) ; passer $=$ mourir $;$ passer $=$ venir chez quelqu'un, éventuellement pour prendre qqch ou cette personne ; passer $=$ cesser $;$ passer $=$ occuper le temps $;$ passer $=$ transmettre $;$ passer $=$ réussir ; Toutes ces catégories sont présentes dans les quatre corpus, avec, là aussi, des contenus variables en mots et en quantités : par exemple un locuteur canadien pense à une passe de hockey alors qu'un Français évoque le volley-ball. Les locuteurs des quatre zones ont également proposé des calembours basés sur l'homophonie passer / passé : nous trouvons dans chaque corpus un présent, un futur et même un composé.

Quelques catégories ne sont représentées que par quelques réactions dans le corpus français, par exemple: passer $=$ frotter (réactions cirer, essuyer, la raclette), ou l'expression passer pour. De plus les expressions figées évoquées par le stimulus ou le contenant sont différentes dans les quatre zones (F : coup de fil, à la casserole, en revue, dessus, un ange passe ; $\mathrm{B}:$ du coq à l'âne, sans me voir, loin ; $\mathrm{S}$ : en revue, muscade.)

Enfin, pour le stimulus noir, nous avons utilisé la méthode la plus populaire, dite de la « Gestalt sémantique» (Караулов, 2000: 194) qui préconise de définir pour chaque champ associatif environ 5-7 sous-ensembles «qui définissent ou nomment un ensemble d'images mentales, les concepts. Ces concepts signalent de la manière la plus générale les liens typiques du référent désigné dans la culture en question par le stimulus, c'est pourquoi on peut utiliser la «Gestalt sémantique» pour la comparaison interculturelle des données associatives» (Марковина, Данилова 2000:119).

Dans le cas de noir (qui pouvait indifféremment être interprété par les participants à l'expérience comme un adjectif ou un substantif) les réactions associatives se sont réparties selon les zones suivantes : 1- couleur (avec une forte préférence pour «blanc » comme 
antonyme); 2- objets de cette couleur ; 3- obscurité, 4- sentiments évoqués ; 5- couleur de peau ;

On peut également déterminer quelques autres sous-zones plus restreintes : 6- habits de couleur noire ; 7- idée liée à la mort, ainsi que les inclassables «allusions textuelles » et jeux de mots, notamment d'assez fréquents « noir c'est noir » ou « Désir ».

Là aussi nous retrouvons sensiblement les mêmes zones pour les quatre sous-ensembles étudiés, avec cependant des différences de contenu. Seuls les interrogés français ont évoqué l'ivresse (réactions comme un Polonais, ivre). De même nous n'avons trouvé des émotions positives que dans le corpus français : positif ou négatif, vie et mort; protection; rassurant etc.

\subsection{Les superconnecteurs}

Une analyse comparée détaillée des données du dictionnaire inverse est encore à faire, cependant nous pouvons ici dès maintenant proposer la comparaison des listes des réactions liées au plus grand nombre de stimuli. Cette liste, désignée par les psycholinguistes russes comme «noyau de la conscience linguistique », est représentative de la structure en réseau du lexique. Certains nœuds de ce réseau sont liés à un grand nombre d'autres, et sont en général eux-mêmes interconnectés. Ce sont les mots les plus importants, les plus riches en connotations, de cette langue (Tableau №8). Pour comparer nous avons introduit la liste mise en évidence par l'expérience menée en 2008-2009 parmi 5500 étudiants pour le français de France (à partir d'une liste de stimuli de 1100 mots) :

Tableau №8. Liste comparative des 25 réactions liées au plus grand nombre de stimuli. Sont indiqués la réaction et le nombre de stimuli qui l'ont provoquée.

\begin{tabular}{|c|c|c|c|c|c|}
\hline & $\begin{array}{l}\mathrm{F} \text { (sur } 100 \\
\text { stimuli) }\end{array}$ & $\begin{array}{l}\text { B (sur } 100 \\
\text { stimuli) }\end{array}$ & $\begin{array}{l}\text { S (sur 100 } \\
\text { stimuli) }\end{array}$ & $\begin{array}{l}\text { C (sur } 100 \\
\text { stimuli) }\end{array}$ & \begin{tabular}{|l} 
F-2010 (sur \\
$1100)$
\end{tabular} \\
\hline 1 & vie 48 & vie 39 & vie 28 & vie 39 & vie 315 \\
\hline 2 & vivre 45 & bien 32 & vivre 27 & bien 32 & amour 245 \\
\hline 3 & être 35 & être 31 & être 24 & amour 29 & homme 193 \\
\hline 4 & bien 32 & vivre 28 & aimer 21 & être 27 & bien 166 \\
\hline 5 & aimer 31 & amour 27 & bon 21 & plaisir 27 & mort 164 \\
\hline 6 & dire 30 & bonheur 26 & amour 20 & beau 25 & bonheur 154 \\
\hline 7 & grand 28 & beau 23 & beau 19 & espoir 24 & travail 154 \\
\hline 8 & beau 27 & grand 22 & bien 18 & joie 24 & joie 153 \\
\hline 9 & amour 27 & temps 21 & grand 18 & bonheur 24 & enfant 152 \\
\hline 10 & espoir 26 & mort 21 & mal 18 & partir 22 & noir 151 \\
\hline 11 & temps 26 & lumière 20 & fort 18 & tout 22 & temps 146 \\
\hline 12 & mal 25 & bleu 20 & savoir 17 & rien 22 & aimer 144 \\
\hline 13 & bonheur 25 & aimer 19 & femme 17 & beauté 22 & mal 144 \\
\hline 14 & enfant 23 & partir 19 & temps 16 & bon 21 & plaisir 144 \\
\hline 15 & penser 22 & dire 18 & partir 16 & mal 21 & droit 142 \\
\hline 16 & homme 22 & plaisir 18 & enfant 16 & savoir 21 & vivre 139 \\
\hline 17 & plaisir 22 & prendre 18 & espoir 16 & enfant 21 & personne 138 \\
\hline 18 & prendre 22 & savoir 18 & liberté 16 & mort 21 & peur 136 \\
\hline 19 & savoir 22 & loin 18 & manger 16 & temps 20 & terre 136 \\
\hline 20 & personne 21 & enfant 17 & homme 15 & penser 20 & fin 135 \\
\hline 21 & libre 21 & espoir 16 & joie 15 & cœur 20 & liberté 134 \\
\hline 22 & joie 20 & faire 16 & penser 15 & vivre 19 & ami 133 \\
\hline 23 & faire 20 & donner 16 & libre 15 & aimer 19 & argent 133 \\
\hline
\end{tabular}




\begin{tabular}{|l|l|l|l|l|l|}
\hline 24 & rien 20 & rester 16 & bonheur 14 & grand 19 & donner 133 \\
\hline 25 & toujours 20 & rêve 16 & plaisir 14 & donner 19 & moi 133 \\
\hline
\end{tabular}

Dans chaque case du tableau sont portés la réaction et le nombre de stimuli pour lesquels cette réaction a été proposée au moins une fois. Par exemple, pour les participants de France, la réaction vie figure dans les champs associatifs de presque la moitié des stimuli de la liste (48), avec une fréquence totale de 369 occurrences. Parmi les locuteurs des autres pays, cette réaction est également celle qui est provoquée par le plus grand nombre de stimuli, dans une proportion moindre, mais avoisinant ou dépassant le tiers des stimuli. C'était aussi le cas pour les étudiants qui ont participé à l'expérience associative en 20082009. La liste des stimuli qui ont provoqué l'apparition de la réaction vie et sa fréquence varient selon les zones étudiées de la francophonie, cependant 18 stimuli sont communs aux quatre ; il s'agit de mort, cœur, amour, vivre, aimer, reprendre, âme, air, jeune, mourir, soleil, corps, donner, ami, comprendre, enfant, gens, devenir (dans l'ordre décroissant du nombre d'occurrences de cette réaction dans le corpus).

\section{Discussion}

Le DENAF a été entrepris notamment pour apporter une réponse la plus objective possible à la question de l'existence d'une «vision du monde» soutenue par la langue, dite également «image linguistique du monde » (ILM) autour de laquelle se déroulent de vifs débats (voir par exemple Pycco 2012 et sa bibliographie) et qui peut être définie ainsi : « La sémantique des unités lexicales comprend un certain nombre de «présomptions », c'est-àdire de représentations du monde non reflétées dans les usages assertifs courants de cette unité et qui passent souvent inaperçus des participants de la communication (présupposés, connotations, composants de base de la signification). $\langle\ldots>$ Au cours de la communication ces présomptions ne sont généralement pas mises en doute, et un emploi de cette unité de langue en contradiction avec elles provoque une réaction négative des communicants $<\ldots>$ C'est précisément de l'ensemble de ces présomptions que se compose l'image (le modèle) linguistique du monde. (Шмелев 2011)». Comme on le voit il s'agit ici uniquement de structure du champ sémantique des mots, et il convient de distinguer l'ILM telle qu'elle est mise en évidence par les dictionnaires d'associations des objets étudiés par la linguistique cognitive (les concepts, le « lexique mental » etc.).

Le but de cette étude était de vérifier si ce que nous avons défini ici comme ILM était lié à la langue (ici le français) ou non. Nous préconisons que l'étude des associations évoquée par les mots est une méthode adéquate et relativement objective pour étudier ce phénomène. Ainsi, si les locuteurs d'une même langue se trouvant dans des conditions sociolinguistiques différentes présentent néanmoins des réactions d'association semblables, alors on peut en conclure que cette image de la langue dépend de la langue donnée. On sait par ailleurs que ces associations sont sensiblement différentes d'une langue à l'autre, ou même à différentes époques d'une même langue (Уфимцева 2013). Toute la difficulté réside dans le degré de précision de notre analyse : comme on le sait, la structure d'un champ associatif comporte une petite quantité d'associations communes à un grand nombre, puis un grand nombre d'associations uniques. Cette structure, comme dans le cas de l'optimiste et du pessimiste devant un verre plein (vide) à moitié, peut être interprétée de deux manières : pour les uns la grande fréquence des premières réactions prouve que la plupart des interviewés réagit de la même manière, ce qui prouve l'existence de l'ILM. Pour les autres le fait qu'une bonne moitié des réactions soient des hapax infirme cette hypothèse. Nous avons vu cependant en 3.1.3. qu'on pouvait analyser plus finement les 
réactions uniques en les regroupant par groupes sémantiques, ce qui permet de confirmer la première hypothèse plutôt que la seconde.

A ce stade de notre étude, nous pouvons affirmer que dans l'ensemble, quand il s'agit des réactions les plus fréquentes, les locuteurs du français des quatre zones étudiées ont tendance à réagir sensiblement de la même manière, ce qui signifierait qu'on peut parler d'une « image linguistique du monde » des locuteurs du français en général. Le fait que ces réactions soient comparables s'explique aussi par le choix des stimuli - il s'agissait de mots très courants de la langue française, partagés de manière identique par tous ses locuteurs.

Nous pensons toutefois que des différences existent dans les détails, ce qu'une analyse plus approfondie devra montrer : comme nous l'avons vu, les fréquences des réactions, même partagées par le plus grand nombre, peuvent varier d'une zone à l'autre, et, ce qui est certain, c'est que si la partition des champs sémantiques en sous-groupes est la même à chaque fois, le contenu de ces subdivisions est différent selon les différents pays. L'impression générale qui se dégage est que l'image proposée par les locuteurs du Canada est sensiblement différente de celle des « Européens » ayant participé à l'expérience.

Cela devra être confirmé ou infirmé par une analyse détaillée des réactions entrant dans le «noyau linguistique de la langue », que nous préférons désigner avec G. Solé par un terme plus technique de «superconnecteurs » (Solé 2005) : une analyse rapide du tableau №8 permet de constater que, dans l'ensemble, les dix premiers mots de la liste sont les mêmes pour les 4 zones étudiées : vie, vivre, être, bien, aimer, grand, beau, amour, espoir, temps. Ces mots se retrouvent également dans l'expérience de 2008, basée pourtant sur une liste de stimuli différente. On peut donc penser que pour l'ensemble de la zone francophone étudiée, ces 10 mots sont interconnectés avec une grande partie du vocabulaire et jouent un rôle fondamental dans la structure en réseau du lexique de la langue. Il reste toutefois à analyser la suite de la liste (au minimum les 100 premiers mots), là où elle diffère d'un pays à l'autre, ce qui pourrait donner des résultats intéressants. Par exemple, dans la liste canadienne la réaction «ici » a un rang assez élevé de 16 stimuli, alors que ce n'est pas le cas des autres pays francophones étudiés. On s'attachera également à l'étude du " recul » de la réaction «ami », qui occupait la $22^{\mathrm{e}}$ place en 2008-2009 et qu'on retrouve à la $35^{\mathrm{e}}$ place dans le corpus canadien, $53^{\mathrm{e}}$ en France, $69^{\mathrm{e}}$ en Belgique et $72^{\mathrm{e}}$ en Suisse, ce qui est particulièrement étonnant à l'époque des réseaux sociaux et des « amis » omniprésents.

Il nous semblerait très intéressant de continuer cette expérience avec l'étude des associations évoquées par les mots chez d'autres locuteurs du français, par exemple en Afrique francophone. Notons pour conclure que cette étude n'étant pas axée sur l'analyse du bilinguisme ou plurilinguisme des participants, nous n'avons pas pris en compte les différentes langues étrangères qu'ils pratiquent, alors que cela a pu introduire un biais dans les réponses. En effet, nous pouvons supposer que la plupart des Suisses, des Belges et des Canadiens ayant pris part à l'expérience sont bilingues, et dans quelques (rares) cas ce bilinguisme apparait dans les réponses (Dieu $\rightarrow$ god, question $\rightarrow$ answer, prendre $\rightarrow$ nehmen etc.). Par ailleurs nous remarquons que très peu de mots n'appartenant pas au registre commun à toute la Francophonie (stuut $\mathrm{B}$ ou cossin $\mathrm{C}$ en réaction à chose) ont été collectés, ce qui est peut-être dû au caractère académique de cette recherche. Menée sur le terrain par un enquêteur local, elle aurait probablement donné d'autres résultats.

Nous sommes également persuadés que l'utilisation des tests d'associations lexicales dans l'étude du bilinguisme (Grabois, 1999) ou l'apprentissage des langues (Meara 2009) représentent également des perspectives prometteuses de notre étude. 


\section{Références bibliographiques}

A frequency dictionary of French: Core vocabulary for learners, (2009) London, New York: Routledge.

Grabois, H., (1999). The convergence of sociocultural theory and cognitive linguistics: Lexical semantics and the L2 acquisition of love, fear, and happiness. Languages of sentiment: Cultural constructions of emotional substrates. Amsterdam: John Benjamins Publishing Company, pp. 201-233.

Henmon V.A.C. (1924) French word book based on a count of 400.000 running words. Madison: University of Wisconsin.

Juilland, A., Brodin D., Davidovich, C. (1970) Frequency Dictionnary of French Words, La Haye, Paris: Mouton.

Kiss, G., Armstrong G., Milroy, R. The Associative Thesaurus of English www.eat.rl.ac.uk.

Meara, P. M. (2009) Connected Words: Word Associations and Second Language Vocabulary Acquisition. Amsterdam: John Benjamins Publishing Company.

Nelson, D. L., McEvoy, C. L., \& Schreiber, T. A. (1998). The University of South Florida word association, rhyme, and word fragment norms. http://www.usf.edu/FreeAssociation.

Raccah, P.-Y. (2014) . Sémantique des points de vue et contraintes sur les possibles argumentatifs. Du sens à la signification. De la signification aux sens, Bruxelles, New York ; Peter Lang ; pp. 277 300

Solé, R. V. et al. (2005). Language Networks: their structure, function and evolution. Trends in Cognitive Sciences, pp. 20-26.

Underhill, J. (2012). Ethnolinguistics and cultural concepts, Truth, Love, Hate and War. Cambridge: Cambridge University Press. Deryle Lonsdale, Yvon le Bras (2009)

Ассоциативный словарь употребительной русской лексики (2011). Воронеж : Истоки.

Гольдин, В. Е., (2010). Концептуальные переменные образа мира по данным ассоциативных словарей , Компьютерная лингвистика и интеллектуальные технологии. Bып. 9 (16). сс. 97-100.

Гольдин, В. Е., Мартьянов, А. О., Сдобнова, А. П. (2009). Электронный русский ассоциативный словарь школьников. Компьютерная лингвистика и интеллектуальные технологии. Вып. 8 (15). С. 69-74.

Гуц, Е.Н. (2004). Ассочиативный словарь подростка. Омск : Изд-во «Вариант-Сибирь».

Дебренн, М. (2010а). Франиузский ассоциативньй словарь Т.1. От стимула к реакиии. Новосибирск: Изд-во Новосибирского ун-та.

Дебренн, М. (2010b). Франиузский ассоциативный словарь T. 2. От реакции к стимулу, Новосибирск: Изд-во Новосибирского ун-та.

Караулов, Ю. Н. (2000). Показатели национального менталитета в ассоциативно-вербальной цепи. Языковое сознание и образ мира., Москва: Ин-т языкознания РАН. сс. 191-206.

Караулов, Ю. Н., Сорокин, Ю. А., Тарасов Е. Ф., Уфимцева, Н. В., Черкасова, Г. А. (19941998). Русский ассоциативный словарь. т..1-6, М.: Институт русского языка РАН.

Марковина, И. Ю., Данилова, Е. В. (2000). Специфика языкового сознания русских и американцев: опыт построения ассоциативного гештальта текстов оригинала и перевода Языковое сознание и образ мира,. - Москва: Ин-т языкознания РАН. сс. 116-132.

Руссо, М. М. (2012). Неогумбольдтианская лингвистика и рамки «языковой картины мира». Лингвистика и методика преподавания иностранных языков. Выпуск №4, сс. 148-176. 
Стернин, И. А, Рудакова, А. В., (2011). Психолингвистическое значение слова и его описание. Saarbrücken: Lambert Academic Publishing.

Уфимцева, Н.В., Черкасова, Г.А., Караулов, Ю.Н, Тарасов, Е.Ф. (2004). Славянский ассоциативный словарь: русский, белорусский, болгарский, украинский. Москва: Институт языкознания РАН.

Уфимцева Н.В. (2013). Системно-целостный принцип и анализ языковой картины мира. Язык, сознание, коммуникаиия. сс. 122-127.

Черкасова, Г. А., (2006). Исследования статистических характеристик ассоциативных словарей. Языковая личность: текст, словарь, образ мира. К 70-летию чл.-корр. РАН Юрия Николаевича Караулова. сс. 378-388.

Шмелев А. Д. (2011). Допустимо ли изучать русский язык? Toronto Slavic Quarterly. №31 